\title{
Analysis of the Influence of Different Fracture Network Structures on the Production of Shale Gas Reservoirs
}

\author{
Ming Yue $\left(\mathbb{D},{ }^{1}\right.$ Xiaohe Huang $\left(\mathbb{D},{ }^{2}\right.$ Fanmin He $\mathbb{D}^{3},{ }^{3}$ Lianzhi Yang $\mathbb{D},{ }^{1}$ Weiyao Zhu $\left(\mathbb{D},{ }^{1}\right.$ \\ and Zhangxin Chen $\mathbb{1 0}^{4}$ \\ ${ }^{1}$ School of Civil and Resources Engineering, University of Science and Technology Beijing, Beijing 100083, China \\ ${ }^{2}$ Innovation Application Institute, Zhejiang Ocean University, Zhoushan 316000, China \\ ${ }^{3}$ Chengdu Surveying Geotechnical Research Institute Co., Ltd. of MCC, Chengdu 610023, China \\ ${ }^{4}$ Chemical and Petroleum Engineering, University of Calgary, Canada \\ Correspondence should be addressed to Lianzhi Yang; ylz_xiaozhu@126.com
}

Received 23 May 2020; Revised 12 October 2020; Accepted 28 November 2020; Published 15 December 2020

Academic Editor: Huazhou Li

Copyright (c) 2020 Ming Yue et al. This is an open access article distributed under the Creative Commons Attribution License, which permits unrestricted use, distribution, and reproduction in any medium, provided the original work is properly cited.

Volume fracturing is a key technology in developing unconventional gas reservoirs that contain nano/micron pores. Different fracture structures exert significantly different effects on shale gas production, and a fracture structure can be learned only in a later part of detection. On the basis of a multiscale gas seepage model considering diffusion, slippage, and desorption effects, a three-dimensional finite element algorithm is developed. Two finite element models for different fracture structures for a shale gas reservoir in the Sichuan Basin are established and studied under the condition of equal fracture volumes. One is a tree-like fracture, and the other is a lattice-like fracture. Their effects on the production of a fracture network structure are studied. Numerical results show that under the same condition of equal volumes, the production of the tree-like fracture is higher than that of the lattice-like fracture in the early development period because the angle between fracture branches and the flow direction plays an important role in the seepage of shale gas. In the middle and later periods, owing to a low flow rate, the production of the two structures is nearly similar. Finally, the lattice-like fracture model is regarded as an example to analyze the factors of shale properties that influence shale gas production. The analysis shows that gas production increases along with the diffusion coefficient and matrix permeability. The increase in permeability leads to a larger increase in production, but the decrease in permeability leads to a smaller decrease in production, indicating that the contribution of shale gas production is mainly fracture. The findings of this study can help better understand the influence of different shapes of fractures on the production in a shale gas reservoir.

\section{Introduction}

Shale gas as a typical unconventional resource is hosted in organic-rich shale reservoirs $[1,2]$. A shale gas reservoir is tight, and its matrix is mainly nano-micro porous $[3,4]$. A flow regime in shale gas reservoirs, which includes not only seepage but also diffusion, slippage, desorption, and absorption, is obviously different from that in conventional reservoirs [5-7]. Moreover, the gas flow in the shale matrix is multiscale and nonlinear. Many theoretical and experimental studies on these multiscale gas flow characteristics of shale reservoirs $[8,9]$ show that the velocity of shale gas in the matrix is so slow that fracturing development methods must be used to increase the production capacity of shale gas reservoirs [10-15].

Given a special shale bedding structure and fragility, volume fracturing is frequently used in the development of shale gas reservoirs, which causes natural cracks to expand into shear slips of brittle rock, forming a complex fracture network with natural and artificial fractures and increasing volume fractures [16-22] to improve the initial production and ultimate recovery. The traditional hydraulic fracturing which 


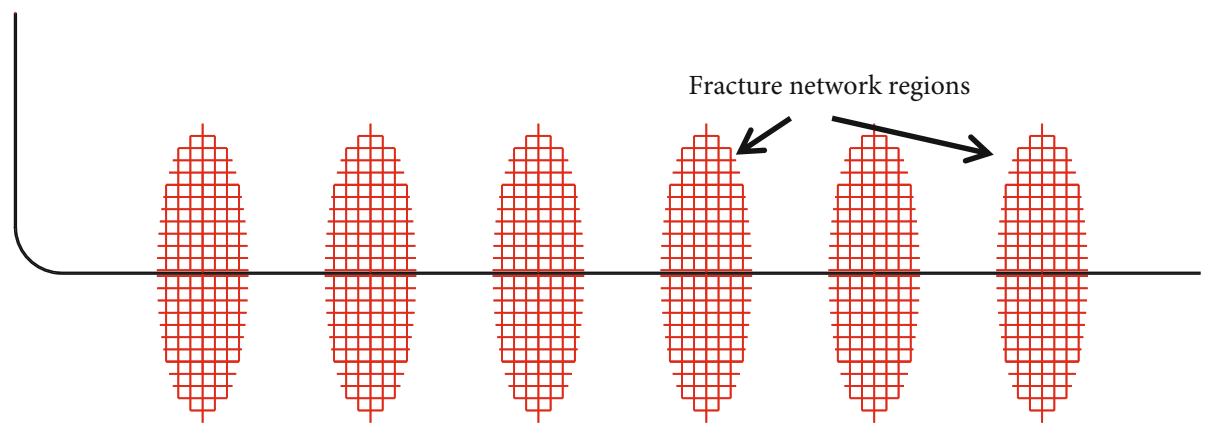

Figure 1: Schematic of fracture distribution of shale gas horizontal wells.

is based on the theory of two symmetrical fractures is inapplicable to volume fracturing networks with natural fractures, bedding, and anisotropic prominent shale gas reservoirs [23-25].

Researching complex fracture networks is very important to have accurate production evaluations for shale gas reservoirs [26, 27]. The two shapes of fracture structures, treelike and lattice-like, have been widely exploited. For shale reservoirs with a tree-like fracture structure, due to difficulty to express the fractures, an equivalent continuous medium model is always used to deal with a fracturing zone through the equivalent permeability according to the fractal theory [28]. For shale reservoirs with a lattice-like fracture network structure, except the treatment of an equivalent continuous medium, a dual-medium model or a discrete fracture network model is also frequently used to simulate seepage characteristics. Eshkalak et al. [29] established a dual-medium model for shale gas with adsorption-desorption and studied the production of single wells through the finite difference method. By using the finite difference method, Swami and Settari [30] determined pressure characteristics and built a production model for gas wells in consideration of the effects of slippage and permeability changes in a fracturing area. Kim et al. [31] considered stress sensitivity and constructed a porous-flow finite element model for shale gas reservoirs with flow-solid coupling. These studies focused only on 2D problems, and no research examined 3D problems. Besides, effects of different fracture network structures on the production of shale gas reservoirs have not been addressed.

The complicated percolation mechanism such as desorption, diffusion, and percolation of shale gas in nanometer porous medium has been studied extensively by scholars, due to the brittleness of shale rock, fracturing is not controllable, and there are just a few laboratory studies on network morphology and seepage law. Zhu et al. [32] established a productivity prediction model of horizontal well multistage fracturing in the shale reservoir considering the interference between the multistage fracturing zones and the pressure drop in the horizontal wellbore. Lu et al. [33] established orthogonal fracture network models with different structures, conducted a series of single-phase and two-phase flow experiments, and studied the influence of seepage characteristics and model structure on permeability in the orthogonal fracture network model. At present, there is a lack of different fracture network morphology description and parameter characterization methods, which cannot better describe or predict the fracture network complexity (density, fracture range, etc.) formed by hydraulic fracturing. Therefore, it is urgent to establish a mathematical model suitable for shale storage characteristics and reveal the gas seepage law under different fracture network conditions.

Numerical methods such as the finite difference method and the finite element method are the powerful methods to study shale gas reservoirs with various fractures [28-36]. First, the finite element algorithm is presented to study multiscale gas flow in a shale reservoir considering desorption, diffusion, and slip effects. Then, the models for the gas reservoir with tree-like and lattice-like fracture structures are established, respectively, under the condition of equal fracture volumes. The flow characteristics of shale gas in a fracturing zone and the effects of fracture network structures on shale gas production are investigated and analyzed. Finally, the factors of shale properties that influence shale gas production in the development process are also researched through the netted fracture models.

\section{Finite Element Model for Seepage of Shale Gas}

For a shale reservoir with micron pores, the seepage of gas can be expressed by the following formula [37]:

$$
C(p) \frac{\partial p}{\partial t}-\left(\kappa_{i j}(p) p_{, i}\right)_{, j}=0
$$

where

$$
\begin{aligned}
\kappa_{i j}(p) & =\frac{\rho(p)}{\mu(p)} k_{i j} K_{0}\left[1+\frac{3 \pi}{16 K_{0}} \frac{\mu D_{\mathrm{k}}}{p}+\frac{b}{4}\left(\frac{3 \pi}{16 K_{0}} \frac{\mu D_{\mathrm{k}}}{p}\right)^{2}\right], \\
C(p) & =\rho_{\mathrm{sc}}\left[\frac{p_{\mathrm{L}} V_{\mathrm{L}}}{\left(p+p_{\mathrm{L}}\right)^{2}}+\frac{T_{\mathrm{sc}} Z_{\mathrm{sc}} p}{p_{\mathrm{sc}} T Z(p)} \phi c_{\mathrm{g}}(p)\right]
\end{aligned}
$$

where $K_{0}$ is the intrinsic permeability, $k_{i j}$ denotes the degree of anisotropy of the matrix when the reservoir is isotropic, $k_{i i}=1.0, k_{i j}=0.0(i \neq j), b$ represents the slippage coefficient, $D_{\mathrm{k}}$ is the Knudsen diffusion coefficient, $\rho$ denotes the density 


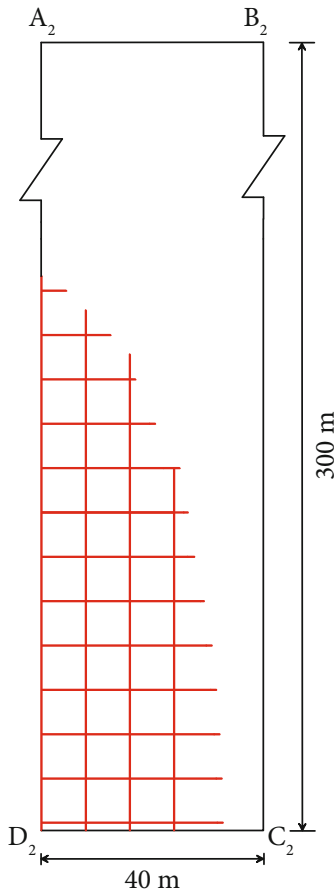

(a) Lattice-like fractures

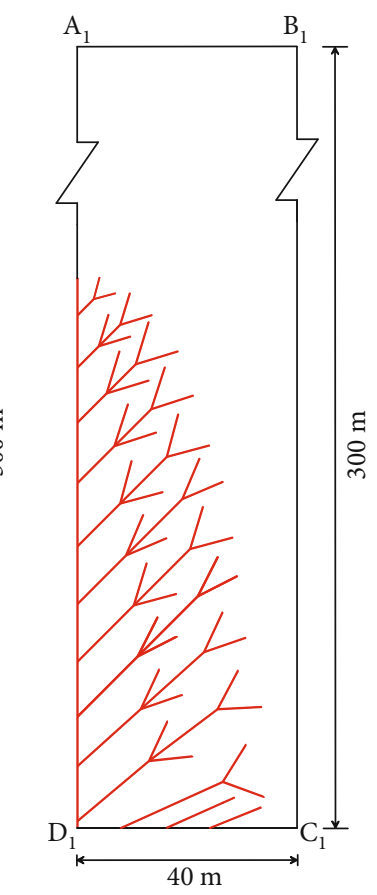

(b) Tree-like fractures

Figure 2: Geometric models of lattice-like and tree-like fractures.

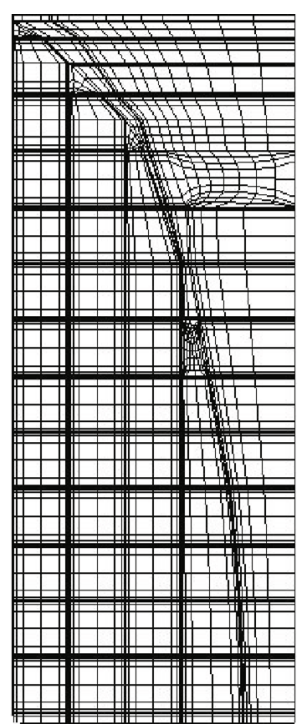

(a) Lattice-like fractures

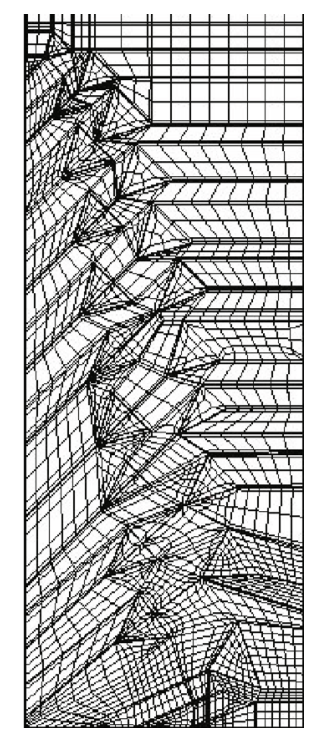

(b) Tree-like fractures

Figure 3: Local area grids of lattice-like and tree-like fracture networks.

of gas, $\phi$ denotes the effective porosity that is assumed unchangeable in this paper, $c_{\mathrm{g}}$ denotes the isothermal compressibility of gas, $p$ denotes the pore pressure, $p_{\mathrm{L}}$ denotes the Langmuir pressure, $V_{\mathrm{L}}$ denotes the Langmuir volume, $\mu$ represents the viscosity coefficient, where the subscript "sc" denotes the variable under standard conditions, $T$ denotes the temperature, and $Z$ denotes the gas compressibility factor, which represents the deviation degree of real and ideal gases under similar conditions.
The finite element method is used to solve Equation (1), and the partial derivative in time in this equation is dealt with an implicit first-order backward difference method. The finite element algorithm for Equation (1) is deduced as

$$
\left[M_{I J}\left(p^{n, m-1}\right)+H\left(p^{n, m-1}\right)\right] p_{J}^{n, m}=H\left(p^{n, m-1}\right) p_{J}^{n-1}+F_{I}\left(p^{n}\right),
$$




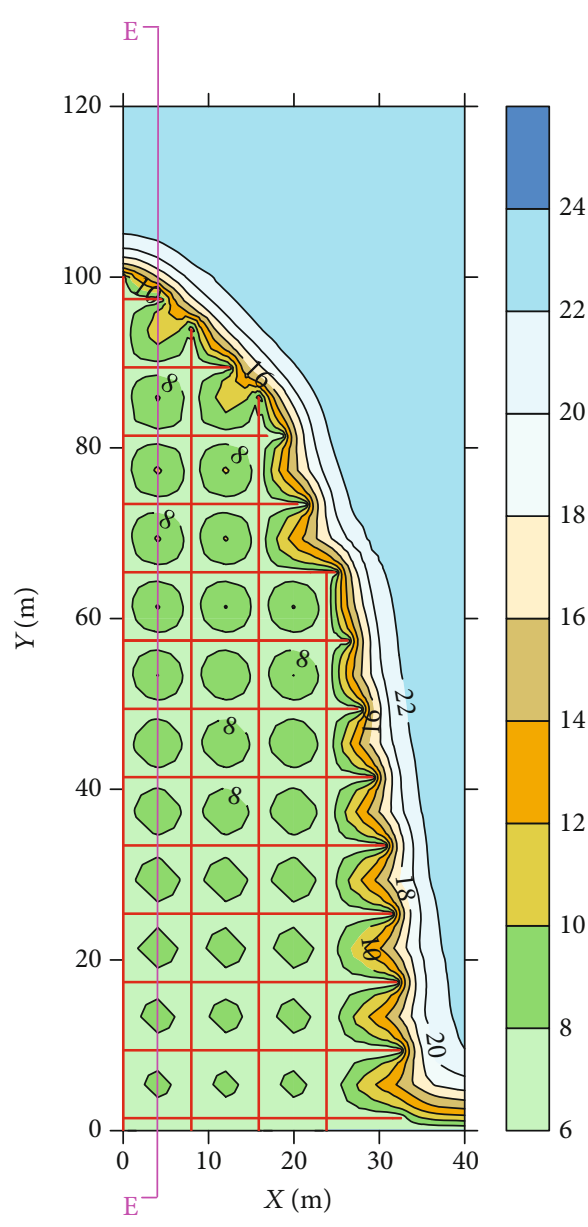

(a) Lattice-like fractures

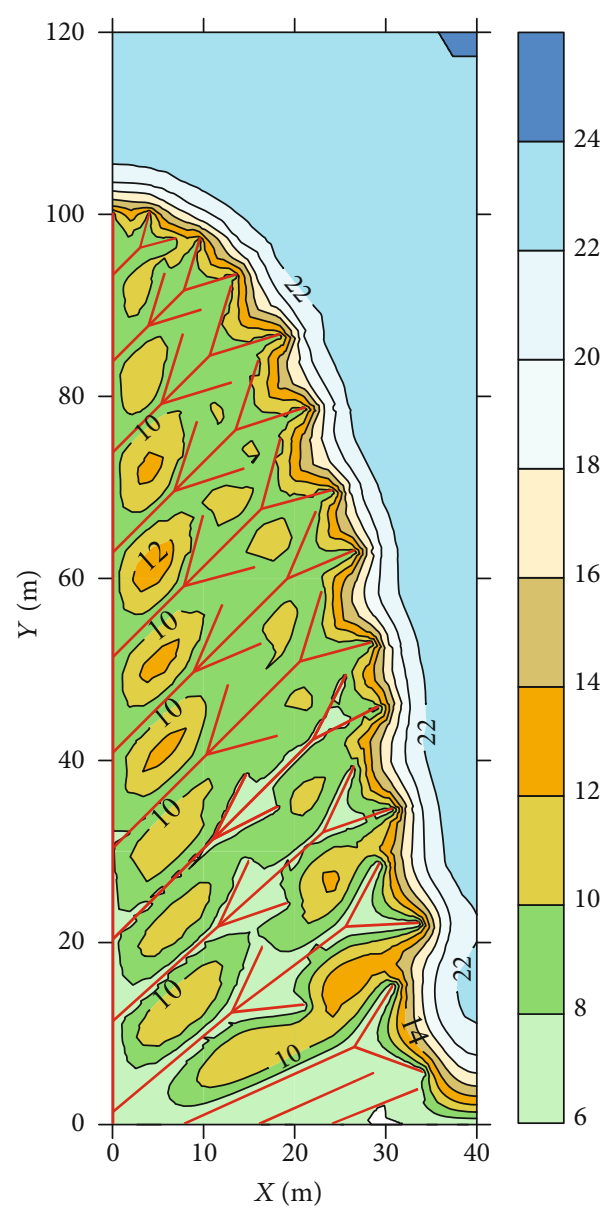

(b) Tree-like fractures

Figure 4: Pressure distribution contour maps after 10 days (unit: MPa).

where

$$
\begin{aligned}
M_{I J}\left(p^{n, m-1}\right) & =\sum_{e} \int_{\Omega_{e}} \kappa_{i j}\left(p^{n, m-1}\right) N_{J, j} N_{I, i} d \Omega, \\
H\left(p^{n, m-1}\right) & =\sum_{e} \int_{\Omega_{e}} \frac{1}{l} \delta_{i j} \frac{C\left(p^{n, m-1}\right)}{t^{n}-t^{n-1}} d \Omega, \\
F_{I}\left(p^{n}\right) & =\oint_{\Gamma} \kappa_{i j} p_{, i} g_{j} N_{I} d L,
\end{aligned}
$$

where $I$ denotes the numbering of nodes in each element $e$, $N_{I}$ denotes the shape function at an internal node of an element, $l$ denotes the total number of nodes in an element, $\delta_{i j}$ denotes the Kronecker delta tensor, $g_{j}$ is the unit outward normal vector at any point of the boundary $\Gamma, n$ denotes the iterative step of time $t(n=0,1,2, \cdots)$, and $m$ denotes the iterative step for solving the seepage field at the time level $t^{n}(m=1,2,3, \cdots)$.

The known pressure boundary (Dirichlet boundary) or the known flux boundary (Neumann boundary) is considered for the initial boundary condition for the nonlinear flow of shale gas.

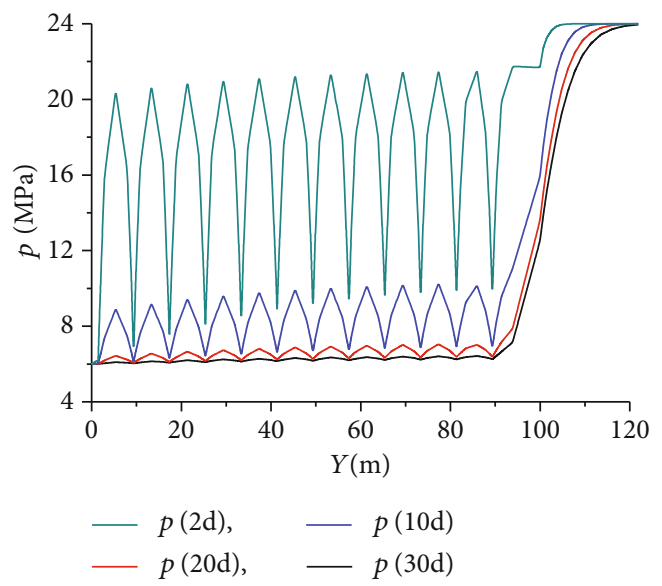

Figure 5: Pressure distribution along line E-E in Figure 4(a) (at the $x=4 \mathrm{~m}$ plane) at different times in the fracture area of the latticelike fracture model (MPa). 


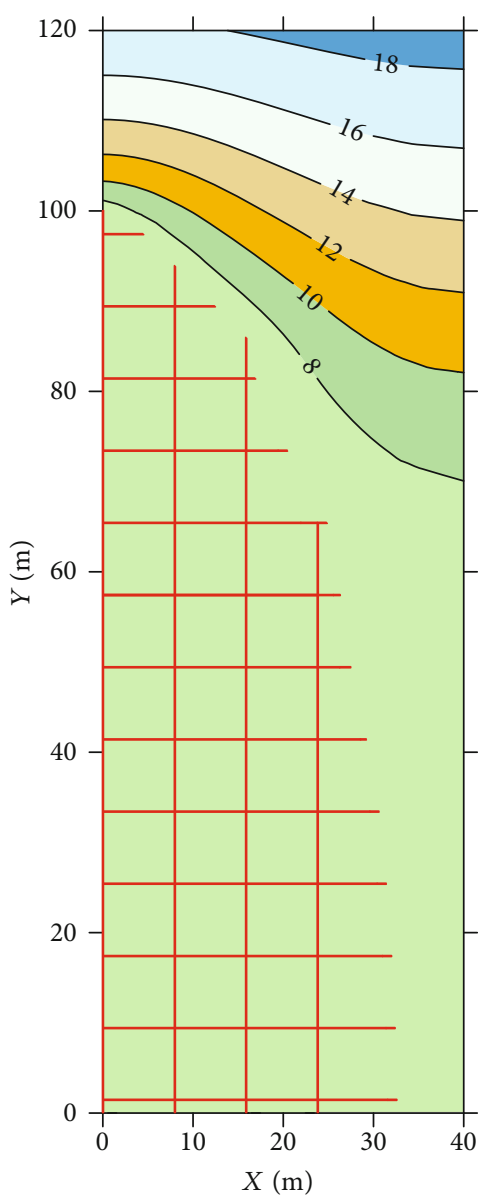

(a) Lattice-like fractures

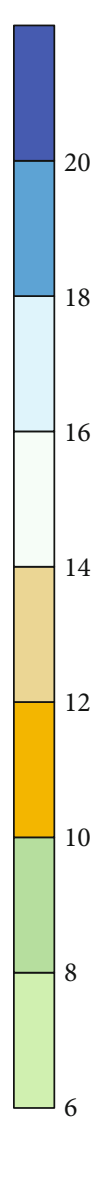

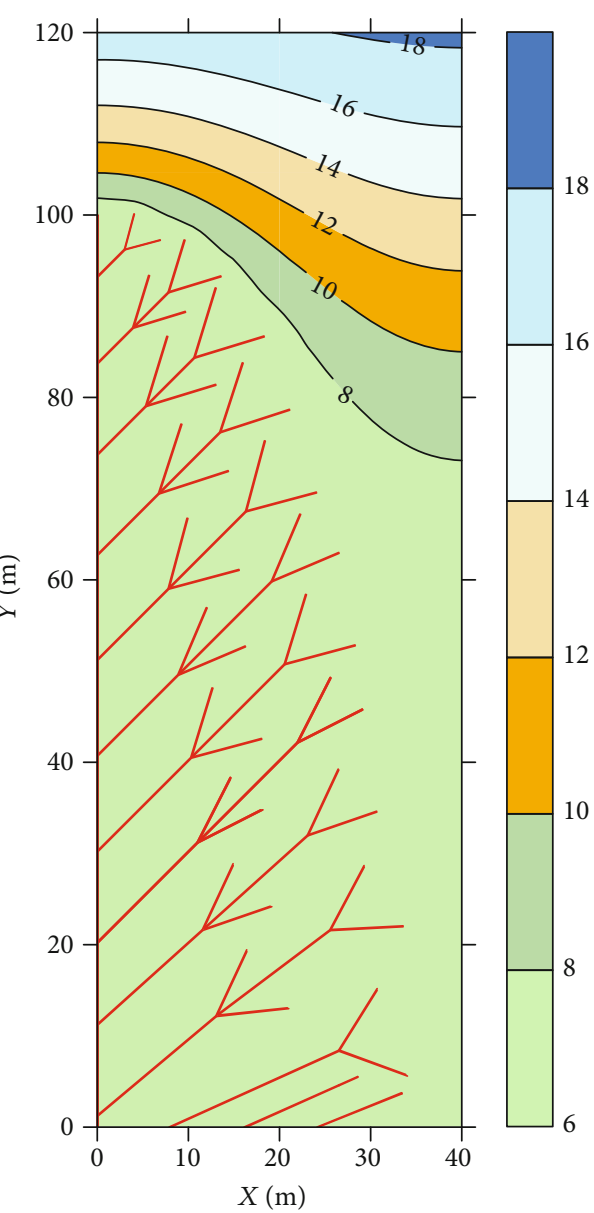

(b) Tree-like fractures

FIgURE 6: Pressure distribution contour map after 600 days (unit: MPa).

(1) The known pressure boundary, in which the pressure value at a node of the boundary is known, does not require an iterative solution. At the boundary, it can be expressed as

$$
\left.p_{I}\right|_{t=t_{0}}=p_{I}^{*}
$$

(2) The known flux boundary can be expressed as

$$
\left.F_{I}(p)\right|_{t=t_{0}}=\oint_{\Gamma}-q^{*}{ }_{n} N_{I} d L,
$$

where $q^{*}{ }_{n}$ denotes the flow velocity on the boundary.

The relationship between pressure and gas viscosity and compression factor can be considered in this finite model. In this model, we use a data table to describe the relationship between pressure and gas viscosity and gas compression factor. But in this study, their relationship is not very important to discuss, so we set up them as constant.

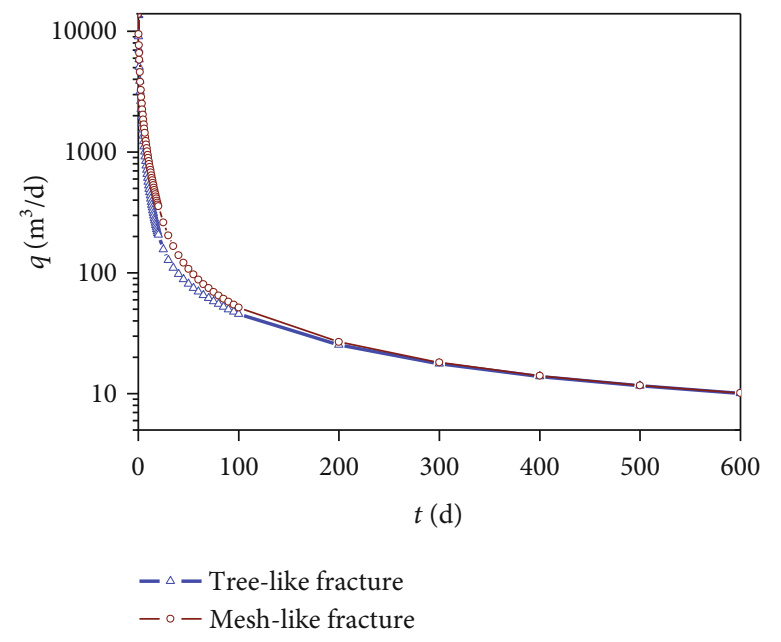

Figure 7: Change in single-day mass flux with time in different fracture networks. 


\section{Shale Gas Production with Different Fracture Structures}

Shale gas is often exploited through horizontal wells and volume fracturing. A schematic of a fracture distribution in shale gas horizontal wells is shown in Figure 1. Many scholars believed that the form of fractures significantly influences the production of shale gas [9]. Lattice-like and tree-like fractures are two typical structures [9]. In this section, 1/4 of a fracturing area is regarded as the research object. The gas flows in the lattice-like and tree-like fractures are investigated below, respectively.

Figures 2(a) and 2(b) show the geometric models for lattice-like and tree-like fracturing regions, respectively. The boundaries of these two regions give equal fracture volumes. The width of the models is $40 \mathrm{~m}$, their height is $300 \mathrm{~m}$, the top of the fracture network elevation is $100 \mathrm{~m}$, and the fracture width is $0.01 \mathrm{~m}$. Lines $A_{1} B_{1}$ and $A_{2} B_{2}$ indicate the outer boundaries, and the pressure values are all $24 \mathrm{MPa}$; lines $\mathrm{C}_{1} \mathrm{D}_{1}$ and $\mathrm{C}_{2} \mathrm{D}_{2}$ indicate the inner boundaries, and the pressure values are $6 \mathrm{MPa}$; lines $\mathrm{A}_{1} \mathrm{D}_{1}$ and $\mathrm{B}_{1} \mathrm{C}_{1}$ and lines $\mathrm{A}_{2} \mathrm{D}_{2}$ and $\mathrm{B}_{2} \mathrm{C}_{2}$ are symmetrical boundaries separately. The thickness of the models is set to $1 \mathrm{~m}$. The parameters of the shale matrix are given as follows:

$$
\begin{aligned}
K_{0 m} & =5 \times 10^{-19} \mathrm{~m}^{2}, \\
D_{\mathrm{k} m} & =9.5 \times 10^{-7} \mathrm{~m}^{2} / \mathrm{s}, \\
b_{m} & =2.425, \\
k_{i i m} & =1.0, \\
k_{i j m} & =0.0(i \neq j), \\
\phi & =0.07, \\
P_{\mathrm{L} m} & =2.5 \mathrm{MPa}, \\
V_{\mathrm{L} m} & =3.74 .
\end{aligned}
$$

The parameters of the fractures which are under the assumption that there is no desorption are as follows:

$$
\begin{aligned}
K_{0 f} & =5 \times 10^{-12} \mathrm{~m}^{2}, \\
D_{\mathrm{k} f} & =0, \\
b_{f} & =0, \\
k_{i i f} & =1.0, \\
k_{i j f} & =0.0(i \neq j), \\
\phi & =0.3, \\
p_{\mathrm{L} f} & =0, \\
V_{\mathrm{L} f} & =0 .
\end{aligned}
$$

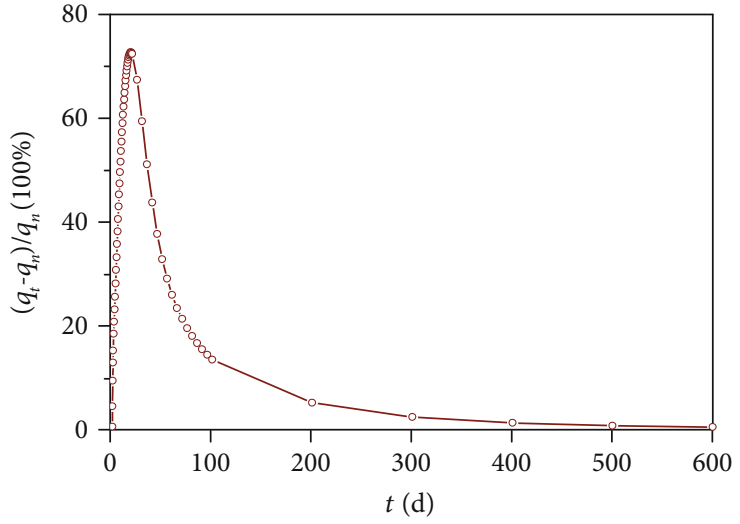

FIGURE 8: Increasing ratio of the flow rate with time for tree-like fractures $q_{t}$ to lattice-like fractures $q_{n}$.

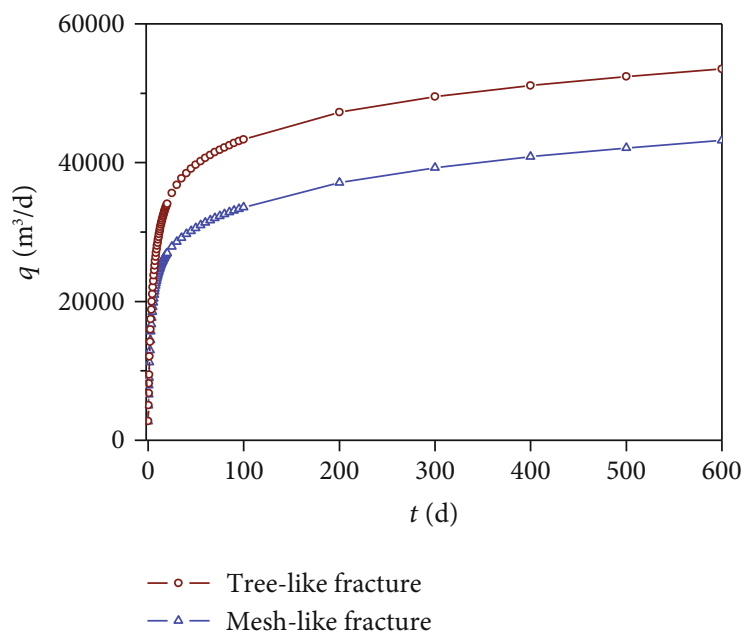

FIgURE 9: Comparison of the cumulative production for lattice-like and tree-like fractures.

The parameters of shale gas in the matrix are set as

$$
\begin{aligned}
\mu & =\text { const }=0.027 \times 10^{-3} \mathrm{~Pa} \cdot \mathrm{s}, \\
z & =\text { const }=0.89 \\
T & =366.15 \mathrm{~K} \\
T & =293 \mathrm{~K} \\
\rho_{\mathrm{sc}} & =0.78 \mathrm{~kg} / \mathrm{m}^{3} \\
\rho_{\mathrm{sc}} & =0.1 \mathrm{MPa}, \\
Z_{\mathrm{sc}} & =1.0
\end{aligned}
$$

The finite element models with the lattice-like and treelike fractures are, respectively, established, and the meshes of the local areas of the fractures are shown in Figure 3. The finite element algorithm in Section 2 is used to calculate the pressure and gas production during the production process.

Figures 4(a) and 4(b) show the pressure distributions in the lattice-like and tree-like fractures after 10 days of 

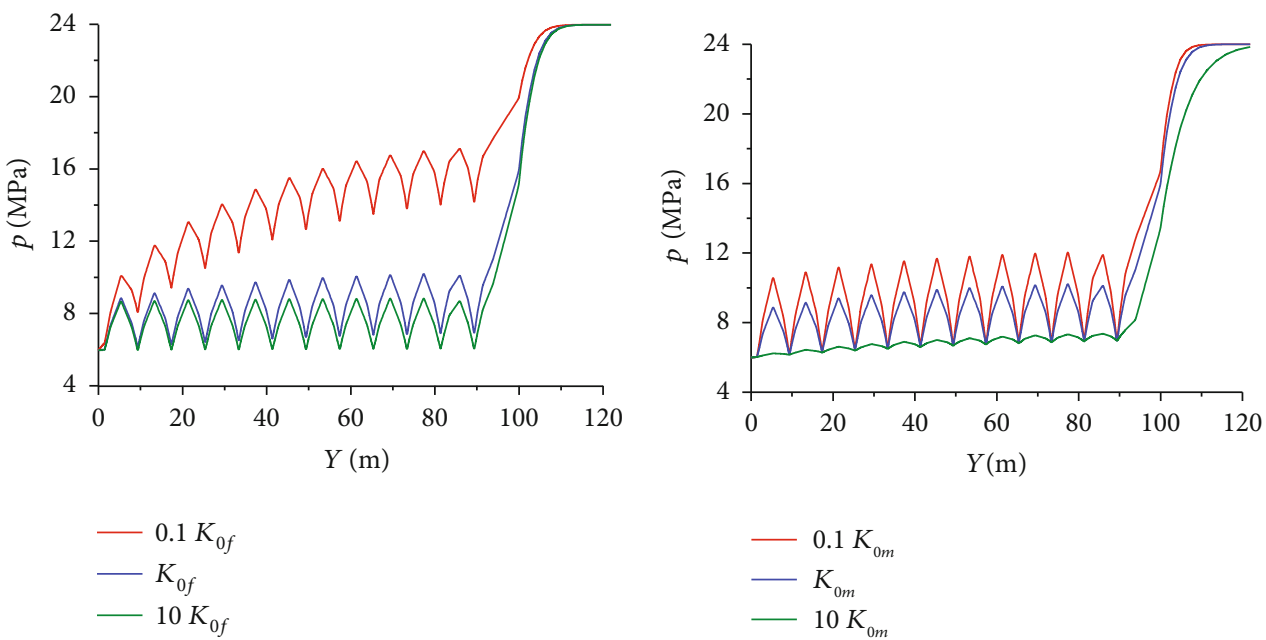

(a) $K_{0 f}$

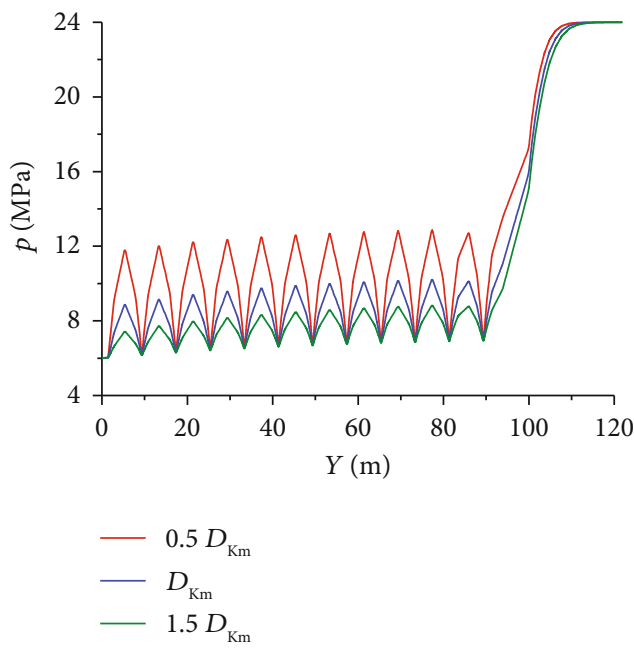

(b) $K_{0 m}$

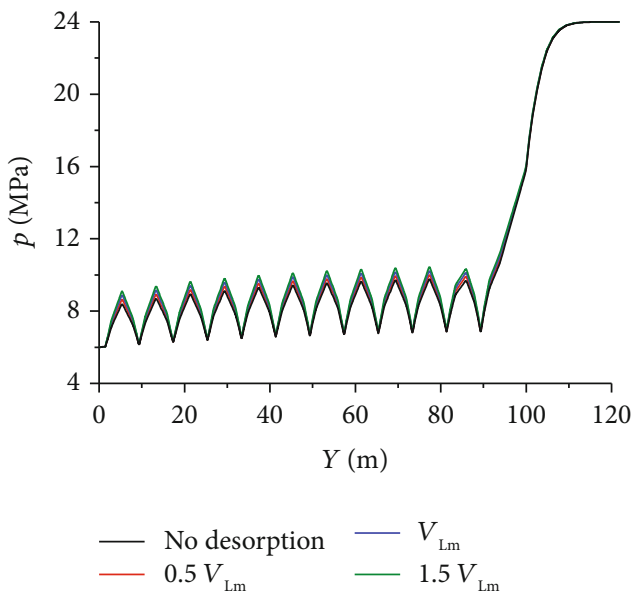

(c) $D_{\mathrm{k} m}$

(d) $V_{\mathrm{L} m}$

FIgURE 10: Pressure distribution of parameters with different values on the cross section $x=4 \mathrm{~m}$ after 10 days of development for the latticelike fractures.

reservoir development, respectively. The internal pressure in the fracture networks releases in both the lattice-like and tree-like fracture models. The change of pressure outside the fracture networks is very small. It can be seen that in the initial production time, the most change of pressure occurs in the fracture networks.

We take line E-E in Figure 4(a) (which is at the $x=4 \mathrm{~m}$ plane) as an example to analyze the pressure distribution. The pressure distributions along this line at different times $(2,10,20$, and $30 \mathrm{~d})$ in the lattice-like fracture model are shown in Figure 5. It is clear that the pressure inside the fracture network is higher than that in the fractures. The pressure in the internal region of the network is gradually released with the passage of time. The initial release is rapid, and the later release is gradual. The pressure release rate outside the network is much lower than that in the fracture network.

Figure 6 presents a pressure distribution diagram after 600 days of development. The pressure field distribution changes in the peripheral fracture network are increased, and the distribution of pressure in the lattice-like and treelike fractures is very similar.

Figures 7 show the distribution maps of single-day mass fluxes with time for the lattice-like and tree-like fracture models, respectively. The changing trend of the flow rate for the lattice-like fracture structure $q_{n}$ is consistent with the flow rate for the tree-like fracture structure $q_{t}$. A clear difference in specific values occurs from 0 to 100 days. With an increase in time, the difference between the two structures decreases. Figure 8 shows an increasing ratio of the flow rate with time for the tree-like fractures $q_{t}$ to the lattice-like fractures $q_{n}$. During early exploitation time, the tree-like fractures' daily production is larger than that of the lattice-like fractures. The largest difference between these two structures occurs on the 20th day. After 300 days, the daily productions of the two fracture structures are almost equal. In the case of the same external boundary conditions and the same area, the structure of fracture networks significantly affects the output of the initial production stage but exerts a minimal 


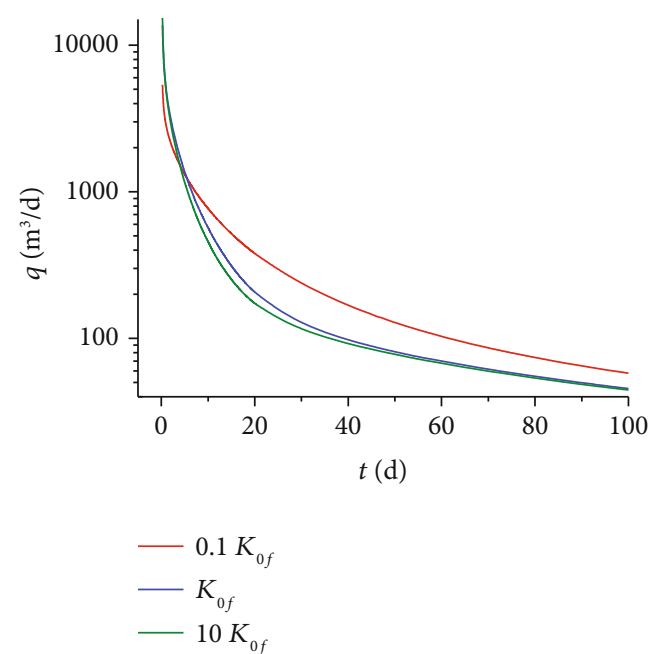

(a) $K_{0 f}$

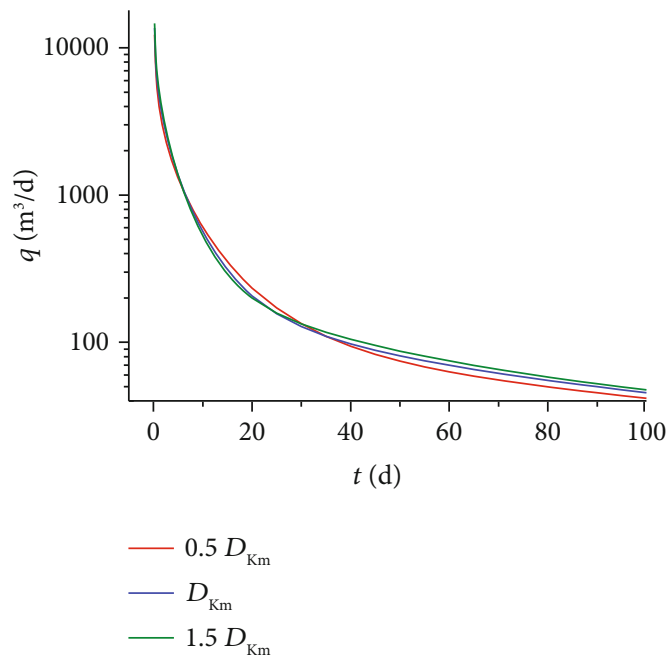

(c) $D_{\mathrm{k} m}$

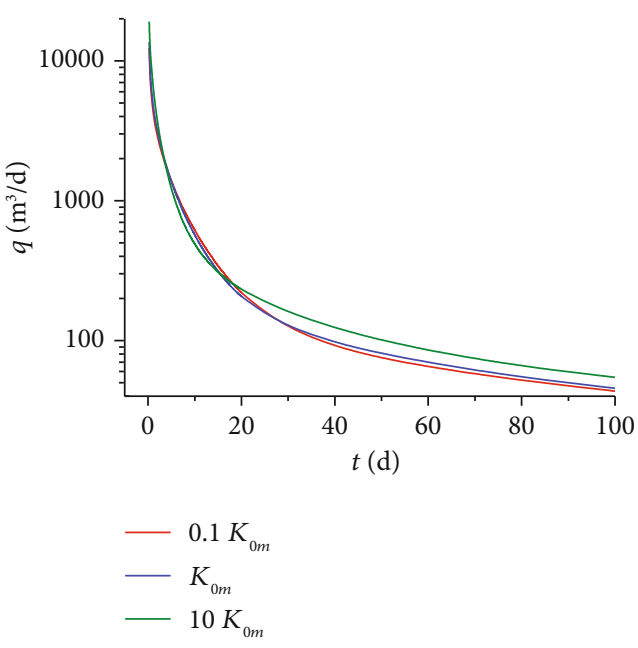

(b) $K_{0 m}$

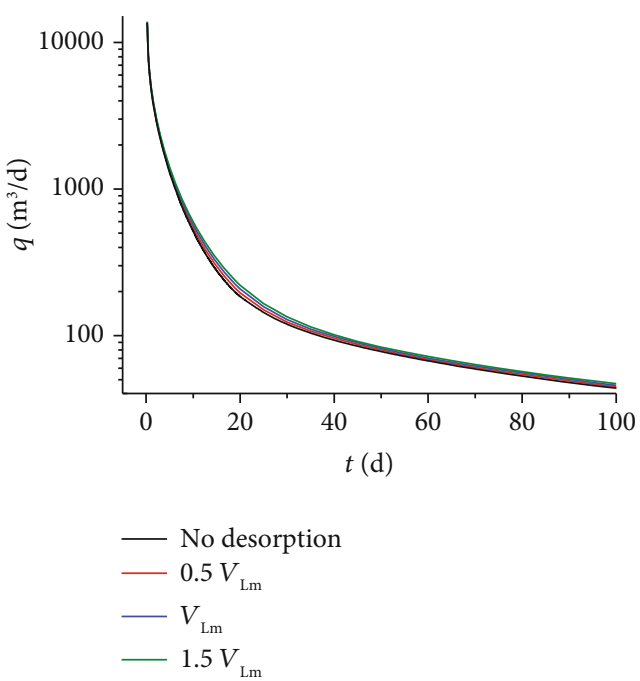

(d) $V_{\mathrm{L} m}$

Figure 11: Change in daily production with different parameters of the lattice-like fractures.

effect on the later period. Figure 9 presents a cumulative comparison of production for the lattice-like and tree-like fractures. The cumulative production of the tree-like fractures is larger than that of the lattice-like fractures. The difference between the two cumulative productions becomes steady after 300 days, which is in accordance with the results shown in Figures 7 and 8.

With the model of the lattice-like fractures as an example, the effects of the permeability of fractures $K_{0 f}$, the intrinsic permeability of the matrix $K_{0 m}$, the diffusion coefficient of the matrix $D_{\mathrm{k} m}$, and the Langmuir volume $V_{\mathrm{L} m}$ on the flow are investigated.

Figure 10 shows the pressure distributions for $K_{0 f}, K_{0 m}$, $D_{\mathrm{k} m}$, and $V_{\mathrm{L} m}$ with different values on the cross section $x=$ $4 \mathrm{~m}$ (line E-E in Figure 4(a)) after 10 days of development for the lattice-like fracture model. $K_{0 f}, K_{0 m}$, and $D_{\mathrm{k} m}$ exert significant influences on the pressure distribution in the vicinity of a fracture network. $V_{\mathrm{L} m}$ exerts a minimal influence on the pressure in the vicinity of the fractures.

Figures 11 and 12 show the production changes with development time under different values of $K_{0 f}, K_{0 m}, D_{\mathrm{k} m}$, and $V_{\mathrm{L} m}$. For $K_{0 f}$, the greater the $K_{0 f}$, the higher the daily production in the early period of development, but in the middle and later periods, a production decline is larger. Figures 11(a) and 12(a) also show that the production will not become larger along with the value of $K_{0 f}$. Thus, the determination of a value of $K_{0 f}$ in a hydraulic fracturing design needs to be combined with reservoir conditions and to be optimized. For $K_{0 m}$ and $D_{\mathrm{k} m}$, the daily and cumulative productions become higher along with the value of $K_{0 m}$ and $D_{\mathrm{km}}$. For $V_{\mathrm{L} m}$, in the early stage of development, due to the existence of fractures, the gas flow in the fracture networks runs very fast, and the influence of $V_{\mathrm{L} m}$ in the matrix on the production is very small; at a later time, because the gas in the fracture networks releases 


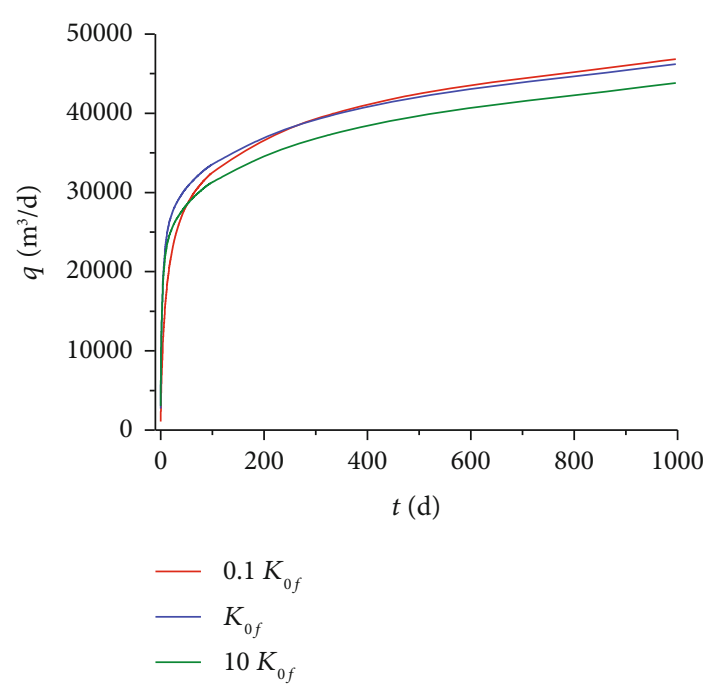

(a) $K_{0 f}$

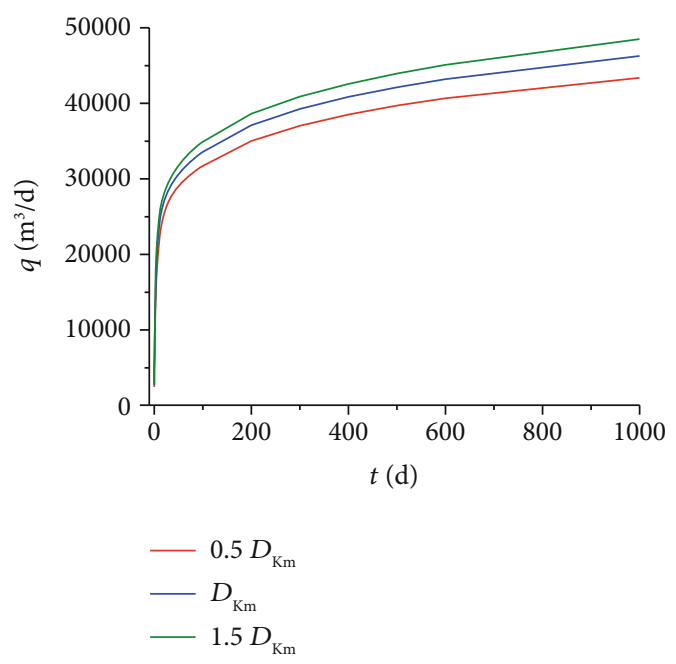

(c) $D_{\mathrm{k} m}$

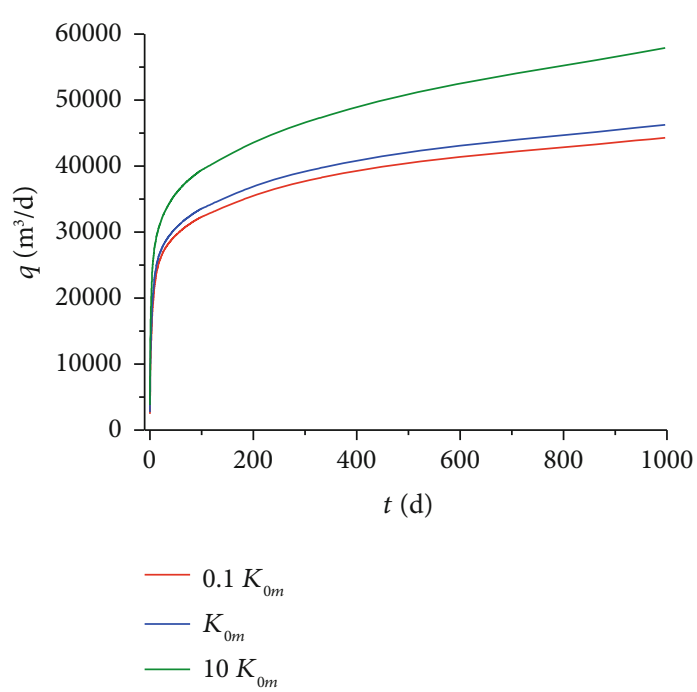

(b) $K_{0 m}$

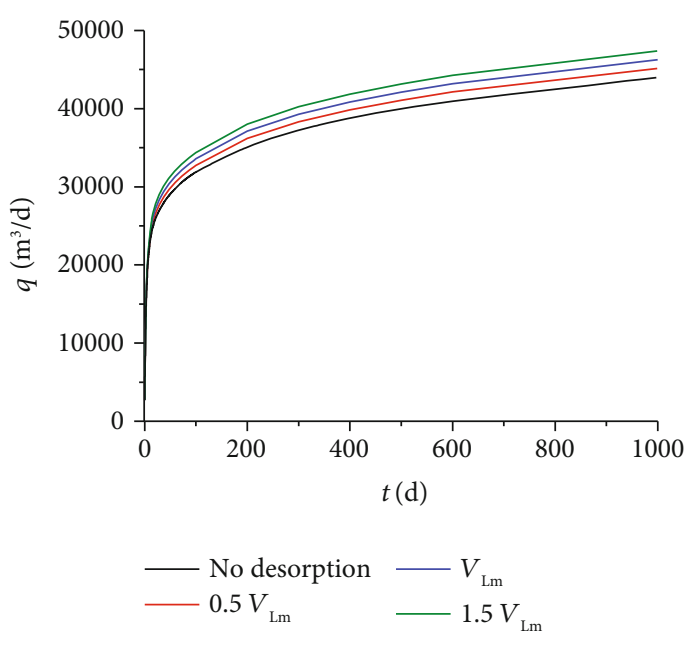

(d) $V_{\mathrm{L} m}$

FIGURE 12: Change in cumulative production with different parameters for the lattice-like fractures.

out, the production mainly comes from the desorption and diffusion effects in the matrix, and the influence of $V_{\mathrm{L} m}$ on the production gradually becomes obvious.

\section{Summary and Conclusions}

Considering the multiscale nonlinear gas seepage theory considering desorption, diffusion, and slip effects of shale, a 3D nonlinear finite element algorithm for shale gas seepage is developed. Finite element models for two different fracture network structures are established under the condition of equal fracture volumes, and the effects on the production of fracture network structures are compared and analyzed.

(1) The early tree-like fracture production is higher than the lattice-like fracture production because the angle between the direction of fracture branches and the flow direction is less than $90^{\circ}$. This angle plays an important role in the seepage of shale gas. Part of the lattice-like fractures perpendicular to the flow direction exerts a minimal effect on the permeability of shale gas reservoirs

(2) In the middle and later periods, owing to a low flow rate in the matrix, the difference between the two fracture network structures can be disregarded, and the productions of these two structures are nearly the same

(3) With the lattice-like fracture model as an example, the influencing factors $\left(K_{0 f}, K_{0 m}, D_{\mathrm{k} m}\right.$, and $\left.V_{\mathrm{L} m}\right)$ for the shale gas production in the development process are analyzed. The Langmuir volume, diffusion coefficient, and matrix permeability are positively correlated with shale gas production, and the matrix permeability exerts a significant influence on production, followed by the gas diffusion coefficient. The production does not become larger 
along with the value of $K_{0 f}$, and the determination of a value of $K_{0 f}$ in a hydraulic fracturing design needs to be combined with the reservoir conditions and to be optimized

(4) The accuracy and efficiency of our numerical method have been shown. Therefore, this method can be used to solve more complicated boundary value problems of shale gas seepage and can be extended to more sophisticated analysis for shale gas reservoirs exploited by fracturing

\section{Nomenclature}

$K_{0}$ : Intrinsic permeability

$k_{i j}$ : Degree of anisotropy of the matrix when the reservoir is isotropic, $k_{i i}=1.0, k_{i j}=0.0,(i \neq j)$

$b$ : Slippage coefficient

$D_{\mathrm{k}}$ : Knudsen diffusion coefficient

$\rho$ : Density of gas

$\phi$ : Effective porosity that is assumed unchangeable in this paper

$c_{\mathrm{g}}: \quad$ Isothermal compressibility of gas

$p$ : $\quad$ Pore pressure

$p_{\mathrm{L}}:$ Langmuir pressure

$V_{\mathrm{L}}$ : Langmuir volume

$\mu$ : Viscosity coefficient, where the subscript "sc" denotes the variable under standard conditions

T: $\quad$ Temperature

$Z$ : $\quad$ Gas compressibility factor

$I$ : $\quad$ Numbering of nodes in each element $e$

$N_{I}$ : Shape function at an internal node of an element

$l$ : $\quad$ Total number of nodes in an element

$\delta_{i j}$ : Kronecker delta tensor

$g_{j}$ : Unit outward normal vector at any point of the boundary $\Gamma$

$n: \quad$ Iterative step of time $t(n=0,1,2, \cdots)$

$m$ : Iterative step for solving the seepage field at the time level $t^{n}(m=1,2,3, \cdots)$

$q_{n}^{*}$ : Flow velocity on the boundary.

\section{Data Availability}

The data used to support the study is available within the article.

\section{Conflicts of Interest}

The authors declare that they have no conflicts of interest.

\section{Acknowledgments}

The work is supported by the National Natural Science Foundation of China (Nos. 51704015 and 11602221) and China Scholarship Council (No. 201706465064).

\section{References}

[1] M. E. Curtis, R. J. Ambrose, and C. H. Sondergeld, "Structural characterization of gas shales on the micro and nano-scales," in Canadian Unconventional Resources and International Petroleum Conference, pp. 1933-1947, Calgary, Alberta, Canada, 2010.

[2] C. N. Zou, R. K. Zhu, B. Bai et al., "First discovery of nano-pore throat in oil and gas reservoir in China and its scientific value," Acta Petrologica Sinica, vol. 27, no. 6, pp. 1857-1864, 2011.

[3] K. Wu, X. Li, C. Wang, W. Yu, and Z. Chen, "Model for surface diffusion of adsorbed gas in nanopores of shale gas reservoirs," Industrial \& Engineering Chemistry Research, vol. 54, no. 12, pp. 3225-3236, 2015.

[4] K. Wu, Z. Chen, X. Li, C. Guo, and M. Wei, "A model for multiple transport mechanisms through nanopores of shale gas reservoirs with real gas effect-adsorption-mechanic coupling," International Journal of Heat and Mass Transfer, vol. 93, pp. 408-426, 2016.

[5] Y. Huang, K. Zhang, Z. Jiang et al., "A cause analysis of the high-content nitrogen and low-content hydrocarbon in shale gas: a case study of the early Cambrian in Xiuwu Basin, Yangtze region," Geofluids, vol. 2019, 13 pages, 2019.

[6] L. He, H. Mei, X. Hu, M. Dejam, Z. Kou, and M. Zhang, "Advanced flowing material balance to determine original gas in place of shale gas considering adsorption hysteresis," SPE Reservoir Evaluation \& Engineering, vol. 22, no. 4, 2019.

[7] M. Wei, Y. Duan, M. Dong, Q. Fang, and M. Dejam, “Transient production decline behavior analysis for a multifractured horizontal well with discrete fracture networks in shale gas reservoirs," Journal of Porous Media, vol. 22, no. 3, pp. 343-361, 2019.

[8] G. Dong, P. Chen, H. Yuan, and Y. Lu, "The experimental investigation of Longmaxi shale dynamic parameters under water-based mud soaking," Geofluids, vol. 2019, Article ID 2128373, 12 pages, 2019.

[9] J. Deng, W. Zhu, and Q. Ma, "A new seepage model for shale gas reservoir and productivity analysis of fractured well," Fuel, vol. 124, pp. 232-240, 2014.

[10] W. Li, S. Yang, D. Yin, L. Lou, J. Guo, and H. Meng, "Development technology and strategy of shale gas," Natural Gas \& Oil, vol. 29, no. 1, 2011.

[11] M. Nassir, A. Settari, and R. G. Wan, "Prediction of stimulated reservoir volume and optimization of fracturing in tight gas and shale with a fully elasto-plastic coupled geomechanical model," SPE Journal, vol. 19, no. 5, pp. 771-785, 2014.

[12] H. Fan, L. Zhang, R. Wang et al., "Investigation on geothermal water reservoir development and utilization with variable temperature regulation: a case study of China," Applied Energy, vol. 275, article 115370, 2020.

[13] J. Wang, H. Song, and Y. Wang, "Investigation on the microflow mechanism of enhanced oil recovery by low-salinity water flooding in carbonate reservoir," Fuel, vol. 266, p. 117156, 2020.

[14] H. Song, J. Xu, J. Fang, Z. Cao, L. Yang, and T. Li, "Potential for mine water disposal in coal seam goaf: investigation of storage coefficients in the Shendong mining area," Journal of Cleaner Production, vol. 244, article 118646, 2020.

[15] M. Yue, Q. Zhang, W. Zhu, L. Zhang, H. Song, and J. Li, "Effects of proppant distribution in fracture networks on horizontal well performance," Journal of Petroleum Science and Engineering, vol. 187, p. 106816, 2020. 
[16] F. Javadpour, D. Fisher, and M. Unsworth, "Nanoscale gas flow in shale sediments," Journal of Canadian Petroleum Technology, vol. 46, no. 10, pp. 55-61, 2007.

[17] G. E. Karniadakis and A. Beskok, Microflows and Nanoflows: Fundamentals and Simulation, Springer, Berlin, 2001.

[18] M. B. Asadi, M. Dejam, and S. Zendehboudi, "Semi-analytical solution for productivity evaluation of a multi-fractured horizontal well in a bounded dual-porosity reservoir," Journal of Hydrology, vol. 581, p. 124288, 2020.

[19] M. Dejam, "Tracer dispersion in a hydraulic fracture with porous walls," Chemical Engineering Research and Design, vol. 150, pp. 169-178, 2019.

[20] M. Dejam, H. Hassanzadeh, and Z. Chen, "Semi-analytical solution for pressure transient analysis of a hydraulically fractured vertical well in a bounded dual-porosity reservoir," Journal of Hydrology, vol. 565, pp. 289-301, 2018.

[21] A. Beskok and G. E. Karniadakis, "Report: a model for flows in channels, pipes, and ducts at micro and nano scales," Microscale Thermophysical Engineering, vol. 3, no. 1, pp. 43-77, 1999.

[22] F. A. Florence, J. A. Rushing, K. E. Newsham, and T. A. Blasingame, "Improved permeability prediction relations for low permeability sands," in Rocky Mountain Oil \& Gas Technology Symposium, pp. 290-307, Denver, Colorado, USA, 2007.

[23] F. Javadpour, "Nanopores and apparent permeability of gas flow in mudrocks (shales and siltstone)," Journal of Canadian Petroleum Technology, vol. 48, no. 8, pp. 16-21, 2009.

[24] S. R. Etminan, F. Javadpour, B. B. Maini, and Z. Chen, "Measurement of gas storage processes in shale and of the molecular diffusion coefficient in kerogen," International Journal of Coal Geology, vol. 123, no. 1148, pp. 10-19, 2014.

[25] D. O. Ezulike and H. Dehghanpour, "A model for simultaneous matrix depletion into natural and hydraulic fracture networks," Journal of Natural Gas Science and Engineering, vol. 16, no. 17, pp. 57-69, 2014.

[26] F. Medeiros, E. Ozkan, and H. Kazemi, "Productivity and drainage area of fractured horizontal wells in tight gas reservoirs," SPE Reservoir Evaluation \& Engineering, vol. 11, no. 5, pp. 902-911, 2013.

[27] L. Mattar, "Production analysis and forecasting of shale gas reservoirs: case history-based approach," in SPE Shale Gas Production Conference, Fort Worth, Texas, USA, 2008.

[28] W. Wang, M. Shahvali, and Y. Su, "A semi-analytical fractal model for production from tight oil reservoirs with hydraulically fractured horizontal wells," Fuel, vol. 158, pp. 612-618, 2015.

[29] M. Eshkalak, U. Aybar, and K. Sepehrnoori, “An integrated reservoir model for unconventional resources, coupling pressure dependent phenomena," in SPE Eastern Regional Meeting, Charleston, WV, USA, 2014.

[30] V. Swami and A. Settari, "A pore scale gas flow model for shale gas reservoir," in SPE Americas Unconventional Resources Conference, Pittsburgh, Pennsylvania, USA, 2012.

[31] J. Kim, E. S. Um, and G. J. Moridis, "Fracture propagation, fluid flow, and geomechanics of water-based hydraulic fracturing in shale gas systems and electromagnetic geophysical monitoring of fluid migration," in SPE Hydraulic Fracturing Technology Conference, The Woodlands, Texas, USA, 2014.

[32] W. Y. Zhu, D. X. Ma, and Q. Qi, "Multi-zone coupling productivity of horizontal well fracturing with complex fracture net- works in shale gas reservoirs," Natural Gas Industry, vol. 37, pp. 60-68, 2017.

[33] Z. G. Lu, J. Yao, and L. F. Li, "Experimental study on fluid flow characteristics in orthogonal fracture network," Journal of China Coal Society, vol. 35, pp. 555-558, 2010.

[34] M. Miller, C. Jenkins, and R. Rai, “Applying innovative production modeling techniques to quantify fracture characteristics, reservoir properties, and well performance in shale gas reservoirs," University of Edinburgh, 2010.

[35] R. Jayakumar, V. Sahai, and A. Boulis, "A better understanding of finite element simulation for shale gas reservoirs through a series of different case histories," in SPE Middle East Unconventional Gas Conference and Exhibition, Muscat, Oman, 2011.

[36] I. Das and M. D. Zoback, "Long period, long duration seismic events during hydraulic fracture stimulation of a shale gas reservoir," in SEG Technical Program Expanded Abstracts 2011, pp. 2011-1473, San Antonio, 2011.

[37] L. Z. Yang, F. M. He, and M. Yue, "A finite-element method for nonlinear seepage flow of shale gas," Scientia Sinica Technologica, vol. 46, pp. 127-134, 2016. 